\title{
Characterization of the Tumor Microenvironment of Neurofibromatosis Type I Plexiform Neurofibromas
}

\author{
Abigail Chmiel ${ }^{1}$, Steven Rhodes ${ }^{2}$, Steve Angus ${ }^{3}$, Yongzheng $\mathrm{He}^{3}$, Quingbo $\mathrm{Lu}^{3}$, Noah Sciaky ${ }^{4}$ \\ Gary Johnson ${ }^{4}$, D. Wade Clapp ${ }^{5}$ \\ ${ }^{1}$ Indiana University School of Medicine; ${ }^{2}$ Riley Children's Hospital Department of Pediatric \\ Hematology/Oncology; ${ }^{3}$ IUSM Herman B Wells Center for Pediatric Research; ${ }^{4}$ UNC Chapel \\ Hill Department of Pharmacology; ${ }^{5}$ Department of Pediatrics, Herman B Wells Center for \\ Pediatric Research, Indiana University School of Medicine
}

\section{Background/Objective:}

Neurofibromatosis type 1 (NF1) is a cancer predisposition syndrome caused by mutations in the NF1 tumor suppressor gene. Patients with NF1 develop tumors of the peripheral nervous system called plexiform neurofibromas (PNs). These histopathologically complex tumors are composed of various immune and inflammatory cells. Mast cells have previously been identified as one key immune cell lineage underpinning PN initiation and progression, however new technologies leveraging RNA-sequencing (RNAseq) allow for the broad and systematic characterization of the PN tumor microenvironment. Here we utilized these tools to delineate PN cellular composition.

\section{Methods:}

RNA seq was performed on murine wild type $(n=6)$ and $P N(n=6)$ tissues. We utilized CIBERSORT to profile the cellular constituents of the PN microenvironment. CIBERSORT is a deconvolution method that uses a reference matrix to estimate the relative proportions of various cell types. Statistical analyses were performed on cell lineage subtypes delineated by CIBERSORT. We further performed a Gene Set Enrichment Analysis (GSEA) to identify which pathways and cytokines might be upregulated in PNs.

\section{Results:}

Using a murine reference matrix, the macrophage lineage, $M 0(p=0.072), M 1(p=0.1)$, were upregulated in PNs $(n=6)$ compared to WT $(n=6)$. A human reference matrix showed M2 $(p=0.025)$ to be upregulated in PNs. GSEA showed IL-1, IL-6, IL-8, TNF and Type I IFN and cytokine secretion to be upregulated in PNs compared to WT.

\section{Conclusion:}

Macrophages were among the most upregulated components of the NF1 tumor microenvironment and upregulation of IL-1, IL-6, IL-8, TNF and Type I IFN production may be contributing to inflammation that is critical in the initiation and progression of PNs.

\section{Scientific/Clinical/Policy Impact and Implications:}

Pharmacotherapies that can target the macrophage lineage and/or aforementioned cytokines may have utility in the treatment of PNs. Further studies are necessary to evaluate this hypothesis. 